\title{
Implantable Venous Access Ports for Chemotherapy in Lung Cancer Patients: Comparison of the Femoral and Subclavian Vein Approaches without Guidance
}

\author{
Takeshi Fujita, Masahiro Tanabe, Masatoshi Kato, Taiga Kobayashi, Etsushi Iida, \\ Naofumi Matsunaga \\ Department of Radiology, Graduate School of Medicine, Yamaguchi University, Yamaguchi, Japan \\ Email: tfujita@yamaguchi-u.ac.jp
}

Received February 15, 2012; revised April 5, 2012; accepted April 25, 2012

\begin{abstract}
Background: The goal of this study was to retrospectively compare the initial success rate and rate of intraoperative and late complications between the femoral and subclavian vein approaches used to implant venous access ports without guidance in lung cancer patients. Methods: We conducted a retrospective review of total 163 lung cancer patients who underwent implantations of a central venous access port for chemotherapy. 95 patients received the ports by the femoral vein blind-puncture technique and 68patients had the port implanted via the subclavian vein blind-puncture technique. The initial success rate of port implantation and the frequency of occurrence of complications were calculated. Results: The primary success rate of venous port implantation was $93.7 \%$ for femoral approach and $88.2 \%$ for the subclavian approach respectively ( $p<0.05$ ). Intraoperative complications developed in two patients $(2.1 \%)$ in the femoral approach group and in five patients (7.4\%) in the subclavian approach group. Although a higher intraoperative complication ratio for the subclavian approach was encountered compared to that for the femoral vein approach, there was no statistically significant difference $(p=0.103)$. Nor was there any statistically significant difference in terms of the occurrence of late complications. Conclusions: Venous access port implantation via the femoral vein approach is safe, and its success rate is very high, with the equal complication rates comparable to the subclavian approach. This approach avoids many of the intraoperative complications. Thus, the femoral vein approach for implanting a venous access port in lung cancer patients should be considered a valid, and safe technique.
\end{abstract}

Keywords: Central Venous Access; Implantable Port; Femoral Vein; Subclavian Vein; Lung Cancer Patients

\section{Introduction}

Long-term central venous access has increasingly become important in the treatment of patients with advanced or unresectable lung cancer undergoing chemotherapy $[1,2]$ because a venotoxic antineoplastic agent may compromise venous integrity, making further treatment impossible. Hickman et al. first introduced the indwelling tunneled externalized central venous catheters, which required daily cleansing of the insertion site and frequent flushing [3]. As totally implantable access ports need no external dressing, allow for normal patient activities, require only monthly flushes of heparinized saline to keep the catheter patent, and are probably have a lower prevalence of septic complications, there has been an increasing demand for such ports among surgeons and oncologists. The central access ports are now being widely used in patients with malignant tumors, and improvements are being made as more patients receive them $[4,5]$.
Currently, conventional subclavian puncture catheterization without guidance is still the predominant method for providing a central venous access port at many institutions [6]. In our institution, we have changed to using a catheter inserted via femoral vein puncture, as the subclavian blind puncture catheterization is associated with the risk of pneumothorax, arterial bleeding, hemothorax, catheter fracture between the clavicle and the first rib, and growth-induced catheter prolapse [7,8]. We herein describe a study comparing the initial success rate and incidence of intra-operative and late complications between the implantation of venous access ports using the femoral versus the subclavian vein approaches without guidance in advanced lung cancer patients.

\section{Materials and Methods}

\subsection{Patients}

We conducted a retrospective review of a total 163 lung 
cancer patients who underwent implantations of a central venous access port for chemotherapy between August 2003 and September 2006 under the care of the same trained interventional radiological team. All procedures for port implantation were performed by three intervenetional radiologists (T F, S Y, and Y W) who had 21, 10, and 7 years of experience, respectively. During the study period, 95 patients (60 men, 35 women,) with a mean age of 58.5 years (range from 38 to 88 years) received a venous port via the femoral vein blind-puncture technique. Of these patients, 70 had non small cell lung cancer (51 Stage III B, 19 Stage IV), 25 had small cell lung cancer (5 Stage II, 10 Stage III, 5 Stage IV). A total of 68 patients (41 men, 27 women) with a mean age of 61.3 years (range from 44 to 86 years) received a port via the subclavian vein blind-puncture technique for port implantation. Of these, 49 patients had non small cell lung cancer (32 Stage III B, 17 Stage IV), 19 had small cell lung cancer (7 Stage II, 6 Stage III, 6 Stage IV). None of the patients were candidates for surgical resection. Histopathological confirmation was performed by the needle biopsy under CT or ultrasound guidance or transbronchial lung biopsy in all patients. Patients with non small cell lung cancer were mainly treated with bi-weekly carboplatin plus paclitaxel, or vinorelbine alone and patients with small cell lung cancer received with cisplatin plus etoposide or irinotecan.

A single port, constructed of silicone rubber (Celsite port, TORAY Medical Industries, Tokyo, Japan) connected to a 5Fr polyurethane catheter (TORAY Anthron PU catheters, TORAY Medical Industries, Tokyo, Japan) was used in all patients in both groups. The region selected for venous access port was based on the patients' requirements. However, in patients with obstruction of the superior vena cava system, enlarged mediastinal lymph nodes, or tumors which could have caused venous compression, the femoral vein approach was chosen. Conversely, the subclavian vein approach was chosen in patients with obstruction of the inferior vena cava system. All patients provided written informed consent before all of the procedures. The study was approved by the local medical ethics committee and was performed in accordance with the Declaration of Helsinki and Good Clinical Practice guidelines.

\subsection{Insertion Technique for Femoral and Subclavian Vein Punctures Catheterization and Venous Port Implantation}

For the femoral procedure, the patient was placed in a supine position on an angiographic table. The skin of the groin (84 right side, 11 left side) and femoral region were prepared with a povidone-iodine-alcohol solution, and $2 \%$ xylocaine was injected subcutaneously for local anesthesia. We used the Seldinder technique to approach the femoral vein. First, the pulsation of the femoral artery was detected manually, and the femoral vein was located about $1 \mathrm{~cm}$ medial to the artery. The femoral vein was punctured subcutaneously with an 18-G hollow needle 2 $\mathrm{cm}$ distal to the inguinal ligament. A Radifocus guidewire (TERUMO Tokyo, Japan) was inserted into the femoral vein through the lumen of the hollow needle, and then the hollow needle was withdrawn. A 5Fr dilator was inserted through the guidewire, then the catheter of the venous port (anthron PU) was inserted into the femoral vein, and the tip of catheter was located immediately below the right atrium of the inferior vena cava by fluoroscopic guidance (Figure 1(a)). A diluted heparin solution was prepared with 3000 IU heparin. The catheter was rinsed with the diluted heparin solution to avoid blood clot formation in the lumen of the catheter. A horizontal incision, about $1 \mathrm{~cm}$ in length, was made on the insertion site. The wound was dissected to the subcutaneous layer on the anterior thigh. A purse-string suture around the insertion site of the catheter was made to prevent blood leakage and subcutaneous hematoma. We used a long blunt-tipped rod to make a vertical subcutaneous tunnel from the puncture site to the anterior femoral region $10 \mathrm{~cm}$ below the groin. The inlet of the tunnel was located lateral to the puncture site, and the distal end of the tunnel was located on the anterior wall of the femoral region. A small horizontal incision, measuring about $2 \mathrm{~cm}$ in length, was made $10 \mathrm{~cm}$ inferior to the inguinal ligament and on the pathway of the subcutaneous tunnel. The catheter was pulled subcutaneously from the inlet to the anterior femoral wound. The pocket for the implantation of the port was created through the anterior femoral incision and then it was positioned at the distal end of the subcutaneous tunnel. The function of the implanted port was tested, and the catheter was fixed at the level of the anterior femoral incision to avoid rotation of the implanted port. The wounds were closed after the port was fixed, and the port was accessed with a 20gauge infusion needle and flashed with $5 \mathrm{ml}$ of heparin solution (Figure 1(b)).

The insertion technique for the subclavian puncture was also performed on the angiographic table. The insertion port was in the right $(n=28)$ or left $(n=40)$ infrasubclavian area from the junction of the outer clavicle to the lowest point of the suprasternal notch. The catheter was inserted in the same way as in the femoral vein puncture without guidance, and was manipulated until the tip of the catheter reached the junction of the superior vena cava and the right atrium as monitored under fluoroscopy. After insertion, a 2-cm transverse incision was made at the anterior line of the axillary between the right 4th and 5th ribs to create a subcutaneous pocket for port implantation. The catheter was lead to the port through a subcutaneous tunnel, and was cut to the appropriate 


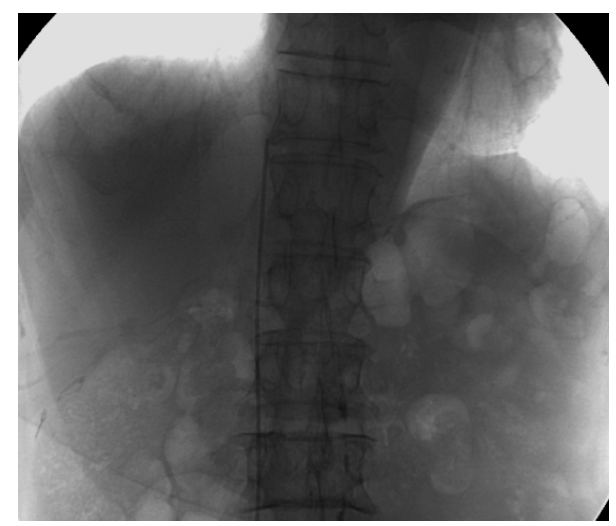

(a)

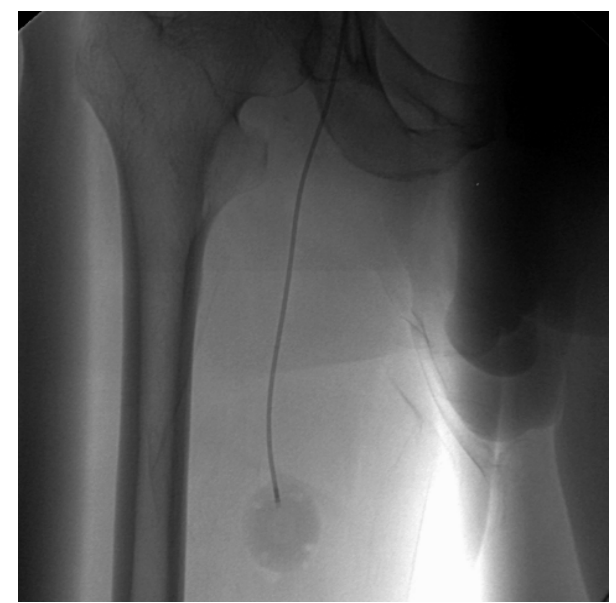

(b)

Figure 1. (a) The tip of catheter was placed manually below the right atrium in the inferior vena cava by fluoroscopic guidance in a 77-year-old man with a non small cell lung cancer; (b). The venous port was implanted at the anterior of the right femoral region about $10 \mathrm{~cm}$ below the inguinal ligament.

length and connected to the port. The procedure of port implantation was the same as that used during the femoral vein puncture catheterization and port implantation. The incision was closed, and the port was accessed with a 20-gauge infusion needle and flashed with $5 \mathrm{ml}$ of heparin solution. Prophylactic antibiotics were not administrated to either group after port implantation (Figure 2).

All patients in this study were followed with routine maintenance at regular intervals until the termination of treatment or the patient's death. Ports were removed if complications resulting in abnormal usage of the port occurred. The initial success rate of port implantation and the frequency of intra-operative and late complications in both groups were calculated. The intra-operative complications included hemothorax, pneumothorax, hematoma, and nerve damage. Late complications include catheter or port infection, aspiration occlusion, catheter pinch-off, venous thrombosis, and catheter migration due

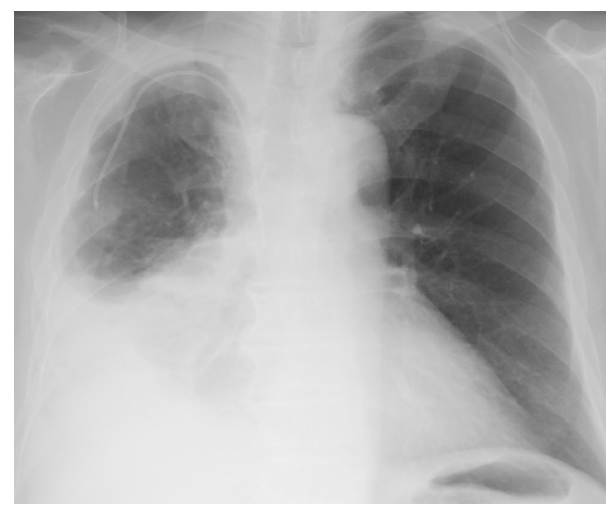

Figure 2. The catheter and venous port was implanted though the right subclavian vein in a 54-year-old man with a non small cell lung cancer.

to spontaneous breakdown of the port. When the primary approach was failed, a contralateral side approach was selected for both the femoral and subclavian approaches.

All data were entered into a computerized data base and were analyzed by using the SPSS statistics medical model for Windows. The chi-squared statistic test or Fisher's exact test were used to establish differences in the distribution of discontinuous variables, and Student's $t$-test or the Mann-Whitney $U$ test were used to compare continuous variable. Tests of significance were two-tailed, and a value of $p<0.05$ was considered to indicate a significant difference.

\section{Results}

The primary success rate of venous port implantation is shown in Table 1. 97.9\% for the femoral approach and $88.2 \%$ for the subclavian approach. This was a statistically significant difference $(p=0.011)$. In the group that received the port via the subclavian approach, the main cause of primary implantation failure was the inability to puncture the subclavian vein $(11.8 \%)$. In contrast, the failure of the femoral venepuncture was mainly due to the presence of a thrombosed femoral vein. This thrombosis in the femoral vein was confirmed by using ultrasonographic examination after all procedures.

A summary of the intraoperative and late complications are also shown in Table 1. Intraoperative complications developed in two patients $(2.1 \%)$ in the femoral approach group and in five patients (7.4\%) in the subclavian approach group. Pneumothorax was encountered in four patients who received the port via a subclavian approach, but no additional treatment was necessary in these patients. Although a higher intraoperative complication ratio was observed for the subclavian approach, the difference was not statistically significant $(p=0.103)$. Late complications developed in 15 (15.8\%) patients who underwent the femoral vein approach, and in 11 (16.2\%) patients in the subclavian approach group. 
Table 1. Success rate and complications according to both port device implantation techniques.

\begin{tabular}{|c|c|c|c|c|}
\hline & & femoral approach $(\mathrm{n}=95)$ & subclavian approach $(\mathrm{n}=68)$ & $p$ value \\
\hline primary success rate(\%) & & 93 (97.9\%) & $60(88.2 \%)$ & 0.011 \\
\hline complication & & 17 (17.9\%) & $16(23.5 \%)$ & 0.377 \\
\hline \multirow[t]{5}{*}{ intraoperative complication } & & $2(2.1 \%)$ & $5(7.4 \%)$ & 0.103 \\
\hline & hemothorax & $0(0 \%)$ & $0(0 \%)$ & \\
\hline & pneumothorax & $0(0 \%)$ & $4(6.2 \%)$ & 0.017 \\
\hline & hematoma & $2(2.1 \%)$ & $1(1.5 \%)$ & 0.766 \\
\hline & nerve damage & $0(0 \%)$ & $0(0 \%)$ & \\
\hline \multirow[t]{6}{*}{ late complication } & & 15 (15.8\%) & $11(16.2 \%)$ & 0.947 \\
\hline & infection & 7 (7.4\%) & $5(7.4 \%)$ & 0.997 \\
\hline & aspiration occlusion & $5(5.3 \%)$ & $3(4.4 \%)$ & 0.804 \\
\hline & pinch-off & $0(0 \%)$ & $1(1.5 \%)$ & 0.236 \\
\hline & venous thrombosis & $3(3.2 \%)$ & $2(2.9 \%)$ & 0.937 \\
\hline & catheter migration & $0(0 \%)$ & $0(0 \%)$ & \\
\hline
\end{tabular}

Catheters and implantable venous ports were removed in 21 patients with infections, catheter occlusion and/or catheter pinch-off. In the other 5 patients, the late complication was primarily venous thrombosis. None of the venous thromboses were large, and none of the patients experienced any symptoms, therefore, the catheter and venous port were not removed. No statistically significant difference was observed between the patients who underwent the femoral versus the subclavian approach in terms of the incidence of late complications. No mortality attributable to the procedure was observed in either group.

\section{Discussion}

The implantation of a venous access port is used universally in patients requiring a permanent device for continuous administration of intravenous medications. Most of these patients are oncology patients included advanced lung cancer, who need to receive chemotherapy or cytotoxic agents $[1,9]$. The venous port device provides safer venous access and a better quality of life than repeated peripheral venous punctures. The most common methods used to implant the venous access port are a percutaneous procedure of the subclavian veins, or the cutdown of the cephalic vein [10-12]. The cephalic vein cutdown method has the advantage of fewer major complications in comparison to the percutaneous subclavian puncture, which can cause hemothorax and pneumothorax [12,13]. However, the cephalic vein cutdown approach can cause difficulties in patients with a small-caliber cephalic vein or venous thrombosis. The inability to locate the cephalic vein or to successfully thread the catheter into the subclavian vein can also reduce the success rate of cephalic cutdown $[11,12]$.
According to the literature, subclavian venous puncture catheterization is recommended as the standard implantable central venous access port, because of its easy and quick manipulation $[1,6,14]$. However, the implantation of a venous access port into the superior vena cava via the subclavian vein may have some disadvantages. For example, the complication rates related to catheterization via the subclavian vein have been described in recent series in the literature, with incidences of up to $2 \%$ for pneumothorax, $14 \%$ for cardiac arrhythmia, 3\% for arterial puncture, and 1\% for serious bleeding [9,15]. In our patients, pneumothorax was reported in 4 cases, and failure to insert the catheter occurred in these patients undergoing a subclavian vein puncture.

Therefore, the higher risk of various intra-operative complications and a lower successful puncture rate in patients with a small-caliber subclavian vein resulted in a switch from using the cephalic or external jugular vein cut down method to the use of catheterization $[10,11]$. The femoral vein approach represents another alternative method of access venous port implantation [16]. The catheter is threaded into the femoral vein using the Seldinger technique, and the port can be implanted in the anterior thigh wall. The possible intra-operative complications of using the femoral route include vascular lesions, lesions of the femoral nerve, formation of hematomas or lymphatic lesions. Although hematomas of the femoral region were observed in two cases, none of the other intra-operative complications were encountered in our series. A low rate of intraoperative complications related to catheterization via the femoral vein approach has also been reported $[16,17]$. However in terms of the incidence of intraoperative complications, the subclavian vein approach was statistically equivalent to the femoral vein approach in our series, although pneumothorax (which 
never occurs in patients undergoing the femoral vein approach) developed in four patients receiving ports via the subclavian approach. Even though experienced interventional radiologists or surgeons perform the puncture, pneumothorax still occurs in a small number of patients [15]. The incidence of pneumothorax as a complication of subclavian vein puncture varies widely, from $2 \%$ to $10 \%[6,11,15]$. Careful attention to anatomic landmarks and operator experience with the insertion of central venous catheters via the subclavian vein are the most important factors for avoidance of intraoperative complications, such as pneumothorax. The majority of pneumothoraces that develop after subclavian vein puncture resolve spontaneously, but in a few intractable cases, the size increases [18]. Especially in patients with pulmonary malignancies included advanced lung cancer, a massive pneumothorax could immediately contribute to the serious conditions, such as respiratory failure and mortality [19]. Even if the experienced surgeons perform the procedure, they may hesitate to puncture the subclavian vein in lung cancer patients with poor respiratory status without image guidance.

Recently, image-guided subclavian puncture using ultrasound or fluoroscopy has been reported to be safe and useful for avoiding intraoperative complications [20,21], however, such technology leads to additional medical costs without any dramatic gains in safety. Therefore, the implantation of a venous access port without image guidance has been performed in the majority of the patients at our institute.

Late complications are of fundamental importance, since these are the main causes of catheter and port removal. The most common late complications were mainly catheter and/or port infection and catheter occlusion $[9,12,14]$. We did not utilize prophylactic antibiotic therapy because there is no clear evidence demonstrating a decrease in the infection rate when it is used [22]. Despite the precaution taken, infection continues to be the main late complication, with bacteremia related to the catheter being the most frequent type. In our series, we observed such complications in 7 (7.4\%) patients, which was similar to what was found by other investigators [16, 23]. There were no statistically significant differences in terms of the rate of late complications such as infection, aspiration occlusion and venous thrombosis between the femoral and subclavian vein approach. Wolosker et al also reported that there were a low rate of late complications in patients who received ports via the femoral approach [16]. Because the port pocket is located near the perineal area, it is presumed that the infection rate for the femoral vein approach would be higher than that using the subclavian vein approach, however no statistically significant difference was found between the two approaches. We speculate that the incidence of catheter or port infection is entirely dependent on keeping the skin around the port clean. Sufficiently sterilizing the skin around the implanted venous port with iodine or alcohol for disinfection is therefore indispensable for preventing contamination via the puncture needle [24]. Skin disinfection with chlorhexidine, or the use of a venous port with a puncture needle coated with antimicrobials have also been shown to be effective by many investigators [24,25].

The other frequent late complications were obstructions of the distal extremity of the catheter and deep vein thrombosis. In the literature, the rates of these complications have been reported to range from 7 to $30 \%$ [26]. In our series, we observed 3 patients who underwent the femoral vein approach, and two patients who underwent the subclavian approach who developed these complications. The clinical suspicion of deep vein thrombosis is commonly based on the presence of edema, pain and erythrocyanosis. Diagnostic confirmation is obtained by duplex scanning. In all of the patients with deep thrombosis in our series, the clinical symptoms were improved by the use of systemic anticoagulation therapy using low molecular weight heparin, and subsequently, warfarin. We do not utilize fibrinolytic treatment because of the inherent risk of bleeding in cancer patients. The conservative treatment should be adopted when the catheter is functioning, allowing the catheter to be successfully maintained $[4,9,26]$.

The catheter and venous port system was removed in all patients with aspiration occlusion of the catheter in both groups. These late complications seemed to be prevented by periodic flushing with heparin diluted with normal saline. A Groshong catheter, which has a twoway valve at the side of the catheter tip, allows blood to be withdrawn and infusion to occur, but can prevent blood from refluxing when the catheter is not. Such a device may be effective for preventing catheter occlusion [27].

Catheter pinch-off between the clavicle and the first rib, which may result in leakage of the chemotherapeutic agent, developed in one case using the subclavian approach [28]. The implantable venous port via femoral vein puncture abolishes the risk of catheter pinch-off.

In addition, a visible "bump" on the chest wall might occur when the port is placed via the subclavian vein. The femoral vein ports are better covered under clothing. This also sometimes can interfere with sleep (Rev 2, Query 3).

There are a few important limitations to this study that should be considered. First, it was a nonrandomized and retrospective study (Rev 1, Query 5) with a small number of patients at a single institute, which may have decreased its statistical strength.

We would speculate that femoral group would have a 
higher complication rate, especially in the infection subcategory if 100 to 200 patients had been observed (Rev 1, Query 2).

Second, there is some selection bias in that the approach was pre-determined from findings on CT scans that indicated that the subclavian approach would be unsuccessful, difficult or would lead to further complications-particularly venous thrombosis (Rev 1, Query 3). Moreover, jugular vein approach under ultrasound guidance is now considered superior to the traditional blind subclavian puncture. If the image guide was available, venous approach under image guidance should be chosen (Rev 1, Query 4, Rev 2, Query 1).

Nevertheless, many operators, particularly surgeons continue to place ports via the subclavian vein, without image guidance. These operators do not use image guidance presumable due to cost considerations or lack of familiarity with direct ultrasound guidance. In this context, our study will be of some value in promoting the femoral vein access as a safer alternative to the subclavian vein access for those operators who do not use image guidance (Rev 2, Query 2).

Third, all of the procedures were performed by only three experienced radiologists; thus the results are biased. Ideally, a randomized multicenter trial should be performed, and our study may provide support for future prospective and randomized investigations in a large number of patients.

In conclusion, the traditional implantation of a venous access port through the subclavian vein is simple and is used throughout the world. However, a percutaneous subclavian puncture without guidance has limitations and risks, including intraoperative complications such as pneumothorax. On the other hand, the low rate of intraoperative complications and superior primary success rate confirms the safety and convenience of using the femoral vein approach. We herein described a safe and effective method for implanting a venous access port through a femoral vein approach without guidance. By avoiding the intraoperative complications associated with the percutaneous approach, the femoral vein approach to implant a venous access port in lung cancer patients can be considered a valid, safe, and suitable alternative when the percutaneous subclavian venous approach is thought to be risky or not optimal.

\section{Acknowledgements}

There was no specific funding for this study, and the authors declare no conflict of interest.

\section{REFERENCES}

[1] E. J. Bow, M. G. Kilpatrick and J. J. Clinch, "Totally Implantable Venous Access Ports Systems for Patients
Receiving Chemotherapy for Solid Tissue Malignancies: A Randomized Controlled Clinical Trial Examining the Safety, Efficacy, Costs, and Impact on Quality of Life," Journal of Clinical Oncology, Vol. 17, No. 4, 1999, p. 1267.

[2] S. Kurul, P. Saip and T. Aydin, “Totally Implantable Venous-Access Ports: Local Problems and Extravasation Injury,” Lancet Oncology, Vol. 3, No. 11, 2002, pp. 684692. doi:10.1016/S1470-2045(02)00905-1

[3] R. O. Hickman, C. D. Buckner, R. A. Clift, J. E. Sanders, P. Stewart and E. D. Thomas, "A Modified Right Atrial Catheter for Access to the Venous System in Marrow Transplant Recipients,” Surg Gynecol Obstet, Vol. 148 No. 6, 1979, pp. 871-875.

[4] J. M. Estes, R. Rocconi, J. M. Straughn, S. Bhoola, C. A. Leath, R. D. Alvarez, L. C. Kilgore and W. K. Huh, "Complications of Indwelling Venous Access Devices in Patients with Gynecologic Malignancies,” Gynecologic Oncology, Vol. 91.No. 3, 2003, pp. 591-595. doi:10.1016/j.ygyno.2003.08.015

[5] R. E. Schwarz, J. S. Groeger and D. G. Coit, "Subcutaneous Implanted Central Venous Access Devices in Cancer Patients: A Prospective Analysis,” Cancer, Vol. 79, No. 8, 1997, pp. 1635-1640.

doi:10.1002/(SICI)1097-0142(19970415)79:8<1635::AID -CNCR30>3.0.CO;2-X

[6] S. Ruesch, B. Walder and M. R. Tramer, "Complications of Central Venous Catheters: Internal Jugular Versus Subclavian Access-Asystematic Review," Critical Care Medicine, Vol. 30, No. 2, 2002, pp.454-460. doi:10.1097/00003246-200202000-00031

[7] L. K. Jablon, K. R. Ugolini and N. C. Nahmias, “Cephalic Vein Cut-Down Verses Percutaneous Access: A Retrospective Study of Complications of Implantable Venous Access Devices,” American Journal of Surgery, Vol. 192, No. 1, 2006, pp. 63-67.

doi:10.1016/j.amjsurg.2005.11.012

[8] P. F. Mansfield, D. C. Hohn, B. D. Fornage, M. A. Gregurich and D. M. Ota, "Complications and Failures of Subclavian-Vein Catheterization,” New England Journal of Medicine, Vol. 331, No. 26, 1994, pp. 1735-1738. doi:10.1056/NEJM199412293312602

[9] C. Ballarini, M. Intra, A. P. Ceretti, A. Cordovana, P. Papani, G. Farina, S. Perrone, M. Tomirotti, A. Scanni and G. P. Spina, "Complications of Subcutaneous Infusion Port in the General Oncology Population,” Oncology, Vol. 56, No. 2, 1999, pp. 97-102.

[10] H. M. Chang, H. F. Hsieh, S. D. Hsu, G. S. Liao, C. H. Lin, C. B. Hsiehand J. C. Yu, "Guidewire Assisted Cephalic Vein Cutdown for Insertion of Totally Implantable Access Ports,” Journal of Surgical Oncology, Vol. 95, No. 2, 2007, pp. 156-157. doi:10.1002/jso.20584

[11] S. P. Povoski, "A Prospective Analysis of the Cephalic Vein Cutdown Approach for Chronic Indwelling Central Venous Access in 100 Consecutive Cancer Patients," Annals of Surgical Oncology, Vol. 7, No. 7, 2000, pp. 496-502. doi:10.1007/s10434-000-0496-9

[12] F. A. D’Angelo, G. Ramacciato, R. A. P De Angelis, P. Amodio, M. Magri and P. Barillari, "Prospective Ran- 
domised Study of Cephalic Vein Cut-Down versus Subclavian Vein Puncture Technique in the Implantation of Subcutaneous Venous Access Devices," Chirurgia Italiana, Vol. 54, No.4, 2002, pp.495-500.

[13] C. M. Seiler, B. E. Frohlich, U. J. Dorsam, P. Kienle, M. W. Buchler and H. P. Knaebel, " Surgical Technique for Totally Implantable Access Ports (TIAP) Needs Improvement: A Multivariate Analysis of 400 Patients,” Journal of Surgical Oncology, Vol. 93, No. 1, 2006, pp. 24-29. doi:10.1002/jso.20410

[14] P. Knebel, L. Fischer, J. Huesing, R. Hennes, M. W. Buchler and C. M. Seiler, "Randomized Clinical Trial of a Modified Seldinger Technique for Open Central Venous Cannulation for Implantable Access Devices,"British Journal of Surgery, Vol. 96, No. 2, 2009, pp. 159-165. doi:10.1002/bjs.6457

[15] R. L. Poorter, F. N. Lauw, W. A. Bemelman, P. J. M. Bakker, C. W. Taat and C. H. N. Veehof, "Complications of an Implantable Venous Access Device (Port-a-Cath) during Intermittent Continuous Infusion of Chemotherapy,” European Journal of Cancer, Vol. 32, No. 13, 1996, pp. 2262-2266. doi:10.1016/S0959-8049(96)00274-2

[16] N. Wolosker, G. Yazbek, M. A. Munia, A. E. Zerati, M. Langer and K. Nishinari, "Totally Implantable Femoral Vein Catheters in Cancer Patients,” European Journal of Surgical Oncology, Vol. 30, No. 7, 2004, pp. 771-775.

[17] S. Y. Chen, C. H. Lin, H. M. Chang, H. M. Hsu and J. C. Yu, "A Safe and Effective Method to Implant a Totally Implantable Access Port in Patients With Synchronous Bilateral Mastectomies: Modified Femoral Vein Approach," Journal of Surgical Oncology, Vol. 98, No. 3, 2008, pp. 197-199. doi:10.1002/jso.21048

[18] L. M. Perlmutt, W. W. Johnston and N. R. Dunnick, "Percutaneous Transthoracic Needle Aspiration: A Review," American Journal of Roentgenology, Vol. 152, No. 3, 1989, pp. 451-455.

[19] P. A. Rubio and E. M. Farrell, "Percutaneous Infraclavicular Subclavian Catheterization," American Journal of Surgery, Vol. 48, No. 5, 1982, pp. 230-231.

[20] S. L. Morris, P. F. Jaques and M. A. Mauro, "RadiologyAssisted Placement of Implantable Subcutaneous Infusion Ports for Long-Term Venous Access," Radiology, Vol. 184, 1992, pp. 149-151.

[21] B. Gebauer, M. El-Sheik, M. Vogt and H. J. Wagner, "Combined Ultrasound and Fluoroscopy Guided Port Catheter Implantation-High Success and Low Complica- tion Rate,” European Journal of Radiology, Vol. 69, No. 3, 2009, pp. 517-522. doi:10.1016/j.ejrad.2007.10.018

[22] P. Y. Marcy, N. Magne, P. Castadot, C. Bailet, J. C. Macchiavello, M. Namer and J. C. Gaard, "Radiological and Surgical Placement of Port Devices: A 4-Year Institutional Analysis of Procedure Performance, Quality of Life and Cost in Breast Cancer Patients," Breast Cancer Research and Treatment, Vol. 92, No. 1, 2005, pp. 61-67. doi:10.1007/s10549-005-1711-y

[23] H. M. Chang, C. B. Hsieh, H. F. Hsieh, T. W. Chen, C. J. Chen, D. C. Chan, J. C. Yu, Y. C. Liu and K. L. Shen, "An Alternative Technique for Totally Implantable Central Venous Access Devices. A Retrospective Study of 1311 Cases,” European Journal of Surgical Oncology, Vol. 32, No. 1, 2006, pp. 90-93.

[24] W. Zingg, A. Imhof, M. Maggiorini, R. Stocker, E. Keller and C. Ruef, "Impact of a Prevention Strategy Targeting Hand Hygiene and Catheter Care on the Incidence of Catheter-Related Bloodstream Infections," Critical Care Medicine, Vol. 37, No. 7, 2009, pp. 2167-2173. doi:10.1097/CCM.0b013e3181a02d8f

[25] D. G. Maki, M. Ringer and C. J. Alvarado, "Prospective randomised Trial of Povidone-Iodine, Alcohol, and Chlorhexidine for Prevention of Infection Associated with Central Venous and Arterial Catheters," Lancet, Vol. 338, No. 8763, 1991, pp. 339-343. doi:10.1016/0140-6736(91)90479-9

[26] A. W. Allen, J. L.Megargell, D. B. Brown, F. C. Lynch, Y. Singh and P. N. Waybill, "Venous Thrombosis Associated with the Placement of Peripherally Inserted Central Catheters," Journal of Vascular and Interventional Radiology, Vol. 11, No. 10, 2000, pp. 1309-1314. doi:10.1016/S1051-0443(07)61307-4

[27] R. Biffi, F. Corrado, F. de Braud, F. de Lucia, D. Scarpa, A. Testori, F. Orsi, M. Bellomi, S. Mauri, M. Aparo and B. Andreoni, "Long Term, Totally Implantable Central Venous Access Ports Connected to Groshong Catheter for Chemotherapy of Solid Tumours: Experience on 178 Cases Using a Single Type of Device,” European Journal of Cancer, Vol. 33, No. 8, 1997, pp. 1190-1194. doi:10.1016/S0959-8049(97)00039-7

[28] D. H. Hinke, D. A. Zandt-Stastny, L. R. Goodman, E. J. Quebbeman, E. A. Krzywda and D. A. Andris, "Pinch-Off Syndrome: A Complication of Implantable Subclavian Venous Access Devices,” Radiology, Vol. 177, No. 2, 1990, pp. 353-356. 